\title{
Fuzzy Set and Multi Descriptions Property
}

\author{
Chee Kau Lim and Chee Seng Chan \\ Centre of Image and Signal Processing \\ Faculty of Computer Science and Information Technology \\ University of Malaya, 50603 Kuala Lumpur, MALAYSIA \\ Email: limck@siswa.um.edu.my, cs.chan@um.edu.my
}

\begin{abstract}
Multi descriptions property is a fundamental property on membership functions of fuzzy sets, which has not been attended so far. The property suggests that not all membership functions of fuzzy sets can be viewed as a simple number in the range of 0 and 1 . In fact, membership function of an element in a fuzzy set should be viewed as the sum of strength of the element that show each attribute which describes the fuzzy set. Therefore, fuzzy sets operation such as union, intersection and subsethood measure need to be revised. By adopting multi descriptions property, this paper introduced a set of improvement based on Checklist Paradigm for these operations for type1 fuzzy sets. Since the multi descriptions property may also appear in type-2 fuzzy sets, we proposed a method for the subsethood measurement, namely Representative Method. This Representative Method provides simple and fast approximate measurements for subsethood.
\end{abstract}

\section{INTRODUCTION}

A membership function is a measure that we use to determine the compatibility of an element in a fuzzy set. Fundamental operations of fuzzy sets such as intersection, union, negation as well as subsethood measurements are the examples of operations on membership functions.

One might believe that the mapping of an element to a fuzzy set with membership function is a linear process, i.e. for any two elements $a_{1}$ and $a_{2}$ in a fuzzy set $A, \mu_{A}\left(a_{1}\right)=\mu_{A}\left(a_{2}\right) \in$ $[0,1]$ represents that the configuration of memberships of $a_{1}$ and $a_{2}$ in $A$ are totally identical. However, this might not be completely correct for the sets that possess multi descriptions property - a property of membership functions suggesting that membership functions may be able to characterized by multiple partially independent attributes. We name these kind of sets as multi descriptions sets.

A membership function may be characterized by multiple partially independent attributes/descriptions. In such cases, a figure that represents the membership function is, in fact come from multiple descriptions that can be "partitioned". Hence, treating a membership function with multi descriptions property like a single number is practically ignored the possible "cavities" in those "partitions", and this brings inaccuracy or incompleteness in computations. Unfortunately, inattention to this multi descriptions property are very common in many fuzzy operations. For example, using min as tnorm in intersection operations, max in as t-conorm in union operations [1]-[3] and Kosko subsethood measurement [4]. In this paper, we discuss this unattended property and propose a novel solution in developing more general fuzzy operations.
We adopted the checklist paradigm [5], which able to generate a list of measurements to find complete solutions for union, intersection and subsethood operations in type-1 fuzzy sets. Nevertheless, for the case of type- 2 fuzzy sets, we propose a novel method on subsethood measurement, namely the Representative Method. This method aims to provide simple, fast, approximate subsethood measurements. While most type2 fuzzy subsethood measurements in the literature [6]-[8] give the results of a measurement as a point value, the Representative Method we proposed gives interval or a type-1 fuzzy set instead, to make the measurements more reliable.

This paper is structured as follow. We discuss the multi descriptions property in Section II, along with the problem that may arise. Two possible solutions of this property are discussed in Section III, with focus are given to the Checklist Paradigm. We extend the solution of subsethood measurement of type-1 fuzzy sets to type-2 fuzzy sets in Section IV, where Representative Method is also discussed. We conclude the paper in Section V.

\section{Multi Descriptions Property of Sets}

It is easier to present the problem with an example in type1 fuzzy sets. Assume that Asian Food, $A$ is a fuzzy set in the universe Food, $X$. An element in this fuzzy set, $x$ is characterized with a membership function $\mu_{A}(x) \in[0,1]$. For a particular food, which denoted as $x^{\prime}$ has $\mu_{A}\left(x^{\prime}\right)=0.8$. My Favourite Food is another fuzzy subset in $X$, denoted $B$. To make the case simpler, assume that $B$ has only 1 element which is exactly $x^{\prime}$. With theorem of union and intersection, we can easily write the following:

$$
\begin{aligned}
& A \bigcup B(x)=\tau^{c}\left(\mu_{A}(x), \mu_{B}(x)\right)=\max \left(\mu_{A}(x), \mu_{B}(x)\right) \\
& A \bigcap B(x)=\tau\left(\mu_{A}(x), \mu_{B}(x)\right)=\min \left(\mu_{A}(x), \mu_{B}(x)\right)
\end{aligned}
$$

where $\tau^{c}$ and $\tau$ are t-conorm and t-norm respectively. We also can find the subsethood of $B$ in $A$ with subsethood theorem proposed by Kosko [4]:

$$
\pi(B \subseteq A)=1-\frac{\sum_{x \in X} \max \left(0, \mu_{B}(x)-\mu_{A}(x)\right)}{\sum_{x \in X} \mu_{B}(x)}
$$


If given that $\mu_{B}\left(x^{\prime}\right)=0.6$, one might take max and min as t-conorm and t-norm respectively to find $A \cup B(x)=$ $\max (0.8,0.6)=0.8, A \bigcap B(x)=\min (0.8,0.6)=0.6$ and $\pi(B \subseteq A)=1-\frac{\max (0,0.6-0.8)}{0.6}=1.0$. Until this stage, we may not see any problem with all these operations. However, if we re-examine these operations closely with considering the nature of membership functions, we will find that there is a flaw in the operations which caused by the multi descriptions property of fuzzy sets.

To explain this flaw, firstly, we should aware that a membership function is a compatibility measure of an element in a fuzzy set. In some cases, this compatibility measure can be quantified easily with the principle of linear increment. For example in the fuzzy set of Tall Man $T$, if $250 \mathrm{~cm}$ is considered absolute tall with $\mu_{T}(250 \mathrm{~cm})=1$ and $\mu_{T}(50 \mathrm{~cm})=0$, so for a particular $t \in[50 \mathrm{~cm}, 250 \mathrm{~cm}]$, the membership function may be assigned as $\mu_{T}(t)=\frac{t-50}{250-50}$. However, this simple assignment of membership functions is only true if the set is a single description set - a set which can be fully specified with only one factor (measurement from head to foot of a standing person in this case).

We used to treat all fuzzy sets as single description sets. In fact, some fuzzy sets are multi descriptions sets, where the membership functions are not determined by a single factor only. For a multi descriptions set, more than one factors are involved in defining a membership function. Each factor, or description $d_{i}$ determines a certain portion of the membership function, such that:

$$
\sum_{i=1}^{N} d_{i}=1
$$

where $N$ is the number of descriptions for the set. All of these descriptions are partially independent. Membership function of an element in a set is given by summing up all the products of descriptions $d_{i}$ and $c_{i} \in[0,1]$, where $c_{i}$ is the strength of the element in showing description $d_{i}$ :

$$
\mu_{A}(x)=\sum_{i=1}^{N} d_{i} c_{i} \in[0,1]
$$

Refer back to the Asian Food example, there is no single attribute that can help us to perfectly identify the membership of a food in $A$. Rather, Asian Food identifies its members with descriptions such as ingredients, method of preparations, cookware to use and etc. Each description contributes certain degree of measure in its membership function. We will not say a food is having high membership degree in set $A$ if it only uses local ingredients, but no other identities of Asian Food can be found.

Since we used to assume all fuzzy sets are single description sets, multi descriptions property of some sets has been ignored. In the calculation of union, intersection and subsethood, we assumed that those membership functions are simple scalar values that we can perform operations such as Eq. (1) -
TABLE I

CONTRIBUTION OF DESCRIPTIONS TO MEMBERSHIP FUNCTIONS OF SET $A$ AND $B$ IN A BEST MATCHING SCENARIOS

\begin{tabular}{l|lllll|l}
\hline Set & $d_{1} c_{1}$ & $d_{2} c_{2}$ & $d_{3} c_{3}$ & $d_{4} c_{4}$ & $d_{5} c_{5}$ & $\mu\left(x^{\prime}\right)$ \\
\hline $\mathrm{A}$ & 0.2 & 0.2 & 0.2 & 0.1 & 0.1 & 0.8 \\
$\mathrm{~B}$ & 0.2 & 0.2 & 0.2 & 0.0 & 0.0 & 0.6 \\
\hline $\mathrm{A} \cap \mathrm{B}\left(x^{\prime}\right)$ & 0.2 & 0.2 & 0.2 & 0.0 & 0.0 & 0.6 \\
$\mathrm{~A} \cup \mathrm{B}\left(x^{\prime}\right)$ & 0.2 & 0.2 & 0.2 & 0.1 & 0.1 & 0.8 \\
\hline
\end{tabular}

(3) directly. In fact, this may not be true. For a multi descriptions set, the membership functions were partitioned by several descriptions as shown in Eq. (5). Simply performing the above operations on membership functions means that we either assume that those sets are single description sets, or it happens that those multi descriptions sets are having membership functions that come from the same descriptions. Both assumptions may bring unexpected results. We illustrate this with the following scenarios.

Let's assume both $A$ and $B$ can be identified with 5 descriptions $d_{i}, i \in\{1,2, \ldots, 5\}$. Each description contributes 0.2 to its membership functions to make the sum 1.0. In some instances, for $x=x^{\prime}$, where $\mu_{A}\left(x^{\prime}\right)=0.8$ and $\mu_{B}\left(x^{\prime}\right)=0.6$, the contribution of each description to the membership function may distribute like what is displayed in Table I, i.e. matrix of the strength of $x^{\prime}$ in showing descriptions corresponding to $d_{1}$ to $d_{5}, c_{1}$ to $c_{5}$ are $[1.0,1.0,1.0,0.5,0.5]$ for $A$ and $[1.0,1.0,1.0,0.0,0.0]$ for $B$.

As we can see in $d_{1} c_{1}$ to $d_{3} c_{3}$, the membership functions of both sets are having maximum overlapping for these descriptions. This is the case that we have assumed so far. While this case represents best matching scenario, taking Eq. (1) for union, Eq. (2) for intersection, as well as Eq. (3) as subsethood measurment will get reasonable results.

In some other instances, matrix of the strength of $x^{\prime}$ in showing descriptions in set $B$ may have the following configuration : $[0.0,0.0,0.2,0.2,0.2]$. In this case, the composition of membership functions may like what we can see in Table II, where membership functions of $x^{\prime}$ for $A$ and $B$ are greatly contributed by different descriptions. Operations that designed to work in best matching scenario are not going to work correctly in this scattered membership function scenario. If we consider the spirit behind the union, intersection and subsethood, we should find that:

- $A \bigcap B\left(x^{\prime}\right)=0.4$ because only 0.2 in $d_{3}$ and 0.1 in both $d_{4}$ and $d_{5}$ are intersected.

- $A \bigcup B\left(x^{\prime}\right)=1.0$ because all the descriptions $d_{1}$ to $d_{5}$ are covered by $A$ or $B$.

- The contribution of membership function of $x$ in $B$ by descriptions $d_{4}$ and $d_{5}$ are greater then membership function of the same element in $A$, so it is clear that $\pi(B \subseteq A)=1$ is not justified anymore.

It is clear that for multi descriptions sets, Eq (1) - (3) only work for the case when the membership functions of both sets have maximum overlapping as presented in Table I. Definitely, this does not represent the general cases where the component of membership functions are scattered to a list of descriptions. 
TABLE II

CONTRIBUTION OF DESCRIPTIONS TO MEMBERSHIP FUNCTIONS OF SET $A$ AND $B$ IN SCATTERED MEMBERSHIP FUNCTION SCENARIO

\begin{tabular}{l|lllll|l}
\hline Set & $d_{1} c_{1}$ & $d_{2} c_{2}$ & $d_{3} c_{3}$ & $d_{4} c_{4}$ & $d_{5} c_{5}$ & $\mu\left(x^{\prime}\right)$ \\
\hline $\mathrm{A}$ & 0.2 & 0.2 & 0.2 & 0.1 & 0.1 & 0.8 \\
$\mathrm{~B}$ & 0.0 & 0.0 & 0.2 & 0.2 & 0.2 & 0.6 \\
\hline $\mathrm{A} \cap \mathrm{B}\left(x^{\prime}\right)$ & 0.0 & 0.0 & 0.2 & 0.1 & 0.1 & 0.4 \\
$\mathrm{~A} \cup \mathrm{B}\left(x^{\prime}\right)$ & 0.2 & 0.2 & 0.2 & 0.2 & 0.2 & 1.0 \\
\hline
\end{tabular}

Adopting multi descriptions property may help us to develop more general and complete expressions for union, intersection and subsethood measurement.

Before we find solutions of multi descriptions property in the next section, we would like to point out that not all fuzzy sets are multi descriptions sets. Set of Expensive Food is an example of classical single description fuzzy set where the membership functions can be completely evaluated with the price of the food. The following are some examples:

- The set of high salary employees is a set with single description, whereas the set of high valued employees is a set with multi descriptions, because the value of an employee may be evaluated with their work ethics, analytical aptitude, ability to learn, etc.

- The set of areas with high transmission rate of H1N1 influenza is a set with single description, whereas the set of people with high risk to H1N1 influenza is a set with multi descriptions property. The age, status of pregnancy, history of diabetes and some other descriptions of a people may determine their risk of being influenced.

- It is worth to point out that some fuzzy sets that may look quite straight forward can be multi descriptions in actual. For example, set of patients with cough may be considered as multi descriptions, because cough has descriptions such as severity, chronic and frequency.

\section{Solutions of Multi Descriptions Property}

\section{A. Type-2 Fuzzy Sets}

Type-2 fuzzy sets (T2FS) theory is an extension of classical fuzzy sets theory, which also called Type-1 fuzzy sets (T1FS) [9]. A T2FS, denoted $\tilde{A}$ is a fuzzy set characterized with type2 membership functions $\mu_{\tilde{A}}(x, u)$, where $x \in X$ and $u$ is the secondary variable such that $u \in J_{x} \subseteq[0,1]$. Each $x_{i} \in X$ has a primary membership $J_{x_{i}}$, which is an interval where $u_{k}, k \in$ $[0,1]$ sits on. The possibility of this secondary variable, $u_{k}$ is given by its secondary grade $f_{x_{i}}\left(u_{k}\right) \in[0,1]$. For more about T2FS please read [10]-[12].

T2FS can be a solution of multi descriptions property up to certain extend, if the original multi descriptions set is a T1FS. This solution can be obtained by developing each of the description of the set into a discrete secondary variable $u$ in T2FS. Hence, the secondary grade $f_{x}(u)$ will become the the strength of an element in showing this description. However, the effort of extending a multi descriptions sets into a higher type is an effort to being precise with all kinds of vagueness. This extension may come with the following shortcomings:
- Solving the problem with a T2FS means we need to develop all the descriptions of the T1FS to a secondary variable in T2FS. The possibility to do so is a challenge if the descriptions of the set is hard to define. "High valued employees" is one of the example where its descriptions and corresponding weights are very subjective.

- After extending the T1FS to T2FS, we may find that some of the descriptions, or secondary variables in T2FS is still describable. For example, "work ethics" as an description of the set "high valued employees" is an expendable description. Do this means that we need to extend the set to a type-3 fuzzy set?

- Again, if the original set is already a T2FS, a higher type fuzzy set is not avoidable in this sense, although the research in this filed is still few and far between.

- Sometime, we may have more then one frameworks to develop the descriptions of a set. Since the descriptions of one framework are not independent from another one, so we need to decide on which framework to choose. For example, Asian Food may be described with details of production (e.g.: ingredients, method of preparations, cookware to use and etc) or the product itself (e.g.: appearance, smell, taste and etc). descriptions of the products, such as taste is always depends on the ingredients and method of preparation (property of methods of production).

- Since each description in a set may have different weight, how to represent this weight is a challenge for a discrete secondary variable.

Thus, T2FS may not be a very good solution for multi descriptions property. In this paper, we propose to adopt the checklist paradigm [5].

\section{B. Checklist Paradigm}

In the case of multi descriptions sets, a general solution for computing membership functions under union, intersection and subsethood operations is not easy to obtain. However, Checklist Paradigm that proposed by Bandler and Kohout [5] may help us to find the bounds and expected values of these operations. Bandler and Kohout have proposed Checklist Paradigm as a solution to generate various kind of logical connectives with bounds. Since Eq. (1) - (3) have been proven in Section II that they only represent the best matching scenario, bounds that included Eq. (1) - (3) are expected to find out through the Checklist Paradigm. For the sake of completeness, we present the Checklist Paradigm here, using fuzzy membership functions instead of crisp value in the original version. To suite our needs, we also replace the checklists with set's descriptions in this presentation.

Consider a list of descriptions $d_{i}, i \in\{1,2, \ldots, \kappa\}$ on both Asian Food $A$ and My Favourite Food $B$. Assume that by studying an arbitrary food $x$, the strength of $x$ in showing each of the description of $A, c_{i}$ can be found. Hence, with Eq (5) we can define membership function of $x$ in $A, \mu_{A}(x)$ as $a=\sum_{i=1}^{\kappa} d_{i} c_{i} \in[0,1]$. Similarly, the membership function of $x$ in $B, \mu_{B}(x)$ can be found too, denoted as $b$. We also 
TABLE III

Checklist Paradigm: The matrix of Relations Between $A$ And $B$

\begin{tabular}{c|c|c|c|}
\multicolumn{1}{c}{} & \multicolumn{1}{c}{$A$} & \multicolumn{1}{c}{$A^{\prime}$} & \multicolumn{1}{c}{ total } \\
\cline { 2 - 4 }$B$ & $s_{00}=\mu_{A \cap B}(x)$ & $s_{10}=\mu_{A^{\prime} \cap B}(x)$ & $b$ \\
\cline { 2 - 4 }$B^{\prime}$ & $s_{01}=\mu_{A \cap B^{\prime}}(x)$ & $s_{11}=\mu_{A^{\prime} \cap B^{\prime}}(x)$ & $1-b$ \\
\cline { 2 - 4 } total & $a$ & $1-a$ & 1 \\
\cline { 2 - 4 } & $a$ &
\end{tabular}

TABLE IV

DEFINING THE RELATIONS OF $A$ AND $B$ IN TERM OF $\theta$

\begin{tabular}{c|c|c|c|}
\multicolumn{1}{c}{} & \multicolumn{1}{c}{$A$} & \multicolumn{1}{c}{$A^{\prime}$} & \multicolumn{1}{c}{ total } \\
\cline { 2 - 4 }$B$ & $\theta$ & $b-\theta$ & $b$ \\
\cline { 2 - 4 }$B^{\prime}$ & $a-\theta$ & $1-a-b+\theta$ & $1-b$ \\
\cline { 2 - 4 } total & $a$ & $1-a$ & 1 \\
\cline { 2 - 4 } & &
\end{tabular}

define $A^{\prime}$ and $B^{\prime}$ as complements of $A$ and $B$ respectively, with membership functions $1-a$ and $1-b$.

By arranging $A, A^{\prime}, B$ and $B^{\prime}$ into a table, we obtain 4 cells that represent $A \cap B(x), A \cap B^{\prime}(x), A^{\prime} \cap B(x)$ and $A^{\prime} \cap B^{\prime}(x)$ (Table III).

Table III can be studied easier if we define the figure in cell $s_{00}$ as $\theta$. This lead to the figure in the other 3 cells being defined (Table IV). Now, it is clear that $\theta$ is the only variable in the table, if $a$ and $b$ has been fixed.

Consider when the value of $\theta$ change but the total in all columns and rows preserved, we can find various extreme configurations for the table. These extreme configurations represent extreme cases of the table and they help us in defining bounds of union, intersection and subsethood.

In two extreme cases, the intersection of $A$ and $B$ are maximum (corresponding to maximum in cell $s_{00}$ or $s_{11}$ ) this can be either when $A^{\prime} \cap B(x)=0$ or $A \cap B^{\prime}(x)=0$. In the first case, $\theta=b$ and in the second case, $\theta=a$. Intersection of $A$ and $B$ also can be expressed as $A$ AND $B$, which corresponding to cell $s_{00}$. In the case $\theta=b, A$ AND $B$ gives $A \cdot B=b$, and in the case $\theta=a$, it gives $A \cdot B=a$. The combination of these two cases gives the definition $A \cap B(x)=\min (a, b)$.

For the union of $A$ and $B$, it can be expressed as $A$ OR $B$. Since $A \cup B=(A \cap B) \cup\left(A \cap B^{\prime}\right) \cup\left(A^{\prime} \cap B\right)$, it is corresponding to cells $s_{00}+s_{01}+s_{10}$. In the case $\theta=b$, it is given by $(b)+(0)+(a-b)=a$; in the case $\theta=a$, it is given by $(a)+(b-a)+(0)=b$. So, this gives the definition $A \bigcup B(x)=$ $\max (a, b)$.

For subsethood, Kohout and Bandler [13] have proposed that fuzzy implication operators measurement:

$$
\pi(A \subseteq B)=\bigwedge_{x \in X}\left(\mu_{A}(x) \rightarrow \mu_{B}(x)\right)
$$

where $\wedge$ can be considered as function min or arithmetic mean, and $\rightarrow$ is fuzzy implication operator defined as NOT $A$ OR $B$ (corresponding to cells $s_{00}+s_{10}+s_{11}$ ). Various valid fuzzy implication operators have been studied in [?], [13], [14]. From Table IV, we can find $(b)+(b-b)+(1-a-b+b)=$ $1-a+b$ and $(a)+(b-a)+(1-a-b+a)=1$ for $\theta=b$ and $\theta=a$ respectively. So, by combining both cases, we get definition:

$$
\pi(A \subseteq B(x))=\min (1,1-a+b)
$$

for the case of one element $x$. For the general case of multiple elements, it is represented by:

$$
\pi(A \subseteq B(x))=\bigwedge_{x \in X} \min (1,1-a+b)
$$

This expression is equivalent to implication under Łukasiewicz implication operator, denoted $I_{€}(a, b)$. Kosko [4] also proved that this expression is equivalent to the subsethood theorem (3) that he derived when the cardinality is normalized.

Using Checklist Paradigm, we found when $A$ and $B$ have maximum intersection, we have min as intersection measure (t-norm), max as union measure (t-conorm) and $I_{\mathrm{E}}$, which is equal to Eq. (3) as subsethood measure. This result is totally identical to the case when the membership functions are in best matching scenario, as presented in Eq. (1) - (3). Since this is in the scenario when $A$ and $B$ has maximum matching, the measurements of intersection and subsethood are the upper bound measurements, whereas the measurement of union is lower bound measurement:

$$
\begin{gathered}
A \bigcup_{\text {low }} B(x)=\max (a, b) \\
A \bigcap_{\mathrm{up}} B(x)=\min (a, b) \\
\pi\left(A \subseteq_{\mathrm{up}} B(x)\right)=\bigwedge_{x \in X} \min (1,1-a+b)
\end{gathered}
$$

In another 2 extreme cases, we have $A \bigcap B(x)=0$ or $A^{\prime} \cap B^{\prime}(x)=0$. The former gives us $\theta=0$ and the later gives us $\theta=a+b-1$. Using these values of $\theta$, we can find another set of measurements, which represent the upper bound of union and lower bounds for both intersection and subsethood:

$$
\begin{gathered}
A \bigcup_{\text {up }} B(x)=\min (1, a+b) \\
A \bigcap_{\text {low }} B(x)=\max (0, a+b-1) \\
\pi\left(A \subseteq_{\text {low }} B(x)\right)=\bigwedge_{x \in X} \max (b, 1-a)
\end{gathered}
$$

This set of expressions gives the upper bound of union as bounded sum t-conorm. The counterpart of this t-conorm, bounded difference $t$-norm was given as the lower bound of intersection. The subsethood measure is given by KleeneDienes fuzzy implication operator, denoted $I_{\mathrm{KD}}$. 
The change of value $\theta$ gives the upper and lower bounds of intersection, union and subsethood. The combination of values reach it expected case when $A \cdot B=a b$, or in another word, $\theta=a b$. In such case, we can find the expected measurements for these operations:

$$
\begin{gathered}
A \bigcup_{\mathrm{ex}} B(x)=a+b-a b \\
A \bigcap_{\mathrm{ex}} B(x)=a b \\
\pi(A \subseteq \text { ex } B(x))=\bigwedge_{x \in X}(1-a+a b)
\end{gathered}
$$

In the expected case, the union and intersection are given by algebraic sum and algebraic product respectively. The subsethood measurement is given by a Reichenbach fuzzy implication operator, denoted $I_{\mathrm{R}}$. This fuzzy implcation operator always gives values sit in between $I_{\mathrm{E}}$ and $I_{\mathrm{KD}}$. One should able to see that in this set of expected value measurements, Eq. (14) will always gives a value greater then Eq. (8) except when at least one of $a$ or $b$ is 0 or 1 . This is to adopt the possibility of the components of membership functions may come from different descriptions of the sets - the scattered membership function scenario. Also due to the scattered membership function scenario, Eq. (15) - (16) will have smaller values compare to Eq. (9) - (10) respectively, unless $a$ or $b$ is(are) 0 or 1 . It is also worth to point out that subsethood measurement using Eq. (16) will not get result 1.0 (represents complete inclusion) unless either $a=0$ or $b=1$. This explained the theorems:

- An empty set is a complete subset of all sets.

- A set is a complete subset in another set if the later set has membership function 1 .

Checklist paradigm generated 3 sets of measurements for union, intersection and subsethood, correspondence to the upper bounds, lower bounds and expected values. In the past, expressions Eq. (8) - (10) are assumed to be the perfect solutions for corresponding operations. But with multi descriptions property, we should learned that those expressions only provide incomplete results, where membership functions are in best matching scenarios. To capture the uncertainties bring by the multi descriptions property, one should consider Eq. (14) - (16), which provide more reliable results. Alternatively, combination of Eq. (8) with Eq. (11), Eq. (12) with Eq. (9) and Eq. (13) with Eq. (10) may also be used if intervals are preferred compare to single-point values.

\section{Subsethood Of Type-2 FuzZy Sets}

Subsethood measurement is an important operation in fuzzy sets theory that may directly relate to applications such as computing with words [3], [15]-[17], fuzzy relational calculus [18], [19] and etc. However, a universally accepted definition of subsethood measurement does not exist. In the past, the authors that have been work on subsethood of T2FS include Nguyen and Kreinovich [6], Rickard et al. [20], Yang and Lin
[7], Zheng et al. [8] and etc. Most of the work mentioned above extends the T1FS subsethood definition of Kosko [21] or its variants, which do not consider the multi descriptions property of fuzzy sets.

All of the above definitions, except Rickard et al [20], give a subsethood measurement as a single-point real number in the interval $[0,1]$. Regarding to this, we would like to do the following deduction: the subsethood measurement of two crisp sets is a boolean; for the measurement of two T1FS, it is a single-point real crisp number in the interval $[0,1]$; thus, for subsethood of two T2FS, it should not be a single-point real number anymore, but an interval or even a T1FS, depends on the type of sets. However, Rickard's subsethood measure for interval T2FS, which produce an interval in $[0,1]$ encounter the problem of low efficiency [20], [22].

In this section, we present a novel method of defining subsethood measurements of T2FS based on fuzzy implication operators. This method, namely Representative Method, produces intervals for the subsethood measurements of interval T2FS, and T1FS as subsethood measurements of T2FS. Since the method is developed based on the fuzzy implication operators derived from Checklist Paradigm, the measurements are robust against multi descriptions property of fuzzy sets.

We illustrate our method in the remaining of this paper with 2 T2FS, namely $\tilde{A}$ and $\tilde{B}$. The secondary variables of these sets are $u$ and $v$ respectively, whereas $f_{x}(u)$ and $g_{x}(v)$ are corresponding secondary grades.

\section{A. Representative Method}

The main objective of Representative Method is to obtain a approximate subsethood measure for 2 T2FS with simple and fast calculations. The strategy to achieve this objective is as follow:

1) For each T2FS $\tilde{A}$ and $\tilde{B}$, find a compatible T1FS that can represent the set in term of its domain in $X$ and its weighing in membership functions;

2) With these compatible T1FS, we perform calculations based on expressions that we retrieved in from Checklist Paradigm for T1FS to obtain the subsethood measurement.

1) Representative Fuzzy Sets: Firstly, we can view a T2FS as a union of multiple elements $x$ over the universe $X$. Each element in the set has its own membership function, which is a T1FS in range $J_{x_{i}} \subseteq\left[\underline{u}_{i}, \bar{u}_{i}\right]$. This T1FS, which also called vertical slice is a two-dimensional plane showing the possibility of each secondary variable, $u_{i}$ in the domain.

As these vertical slices are T1FS, we can perform defuzzification to find each a point-value that represents the whole $\mathrm{T}_{1 F S}{ }^{1}$. Center-of-area defuzzification is a widely used and reasonable method to find the center-of-area as a representation of the T1FS in some fuzzy logic systems [1], [25], [26].

Center-of-area of a T1FS also called centroid. It divides a graph of membership function of a T1FS into two portions

\footnotetext{
${ }^{1}$ In some fuzzy logic systems [23], [24], a defuzzification procedure is used to obtain deterministic output action after the inferencing procedure. However, we use the same method here to perform type-reduction on input.
} 


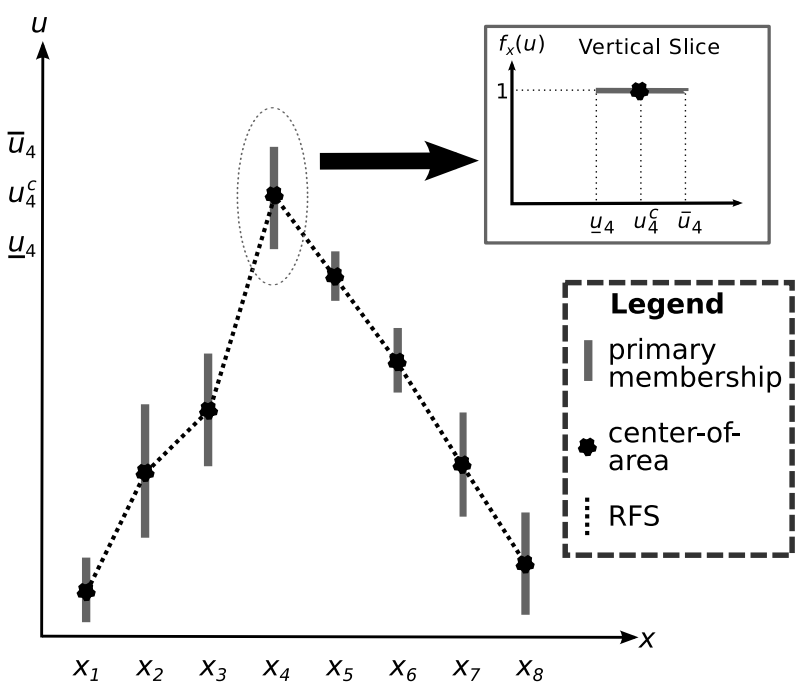

Fig. 1. Once the center-of-area of all the elements are obtained, we can just link them up to form a representative fuzzy set, RFS. The upper right insert is the vertical slice of one of the membership function. For interval T2FS, the center of sets are always the middle point of upper and lower bounds of the interval.

with equal size. In case of a T2FS $\tilde{A}$, the center-of-area of the continuous vertical slice of an element $x$ is $u^{c} \in J_{x}$ such that:

$$
u^{c}=\sum_{u \in J_{x}} u \cdot f_{x}(u) / \sum_{u \in J_{x}} f_{x}(u)
$$

If there is no value in $J_{x}$ match the $u^{c}$, the closest $u$ will be considered as $u^{c}$. For a continuous vertical slice, the symbol of sum can always be replaced with integration $\int$. The corresponding membership function is given by $f_{x}\left(u^{c}\right)$. Applying Eq. (17) on all the vertical slices of a descritized T2FS $\tilde{A}$, we can get each element in $\tilde{A}$ a representative, which is its center-of-area. We can link up all the center-of-areas in the series and find a fuzzy set that represents $\tilde{A}$ on element level (Figure 1). We call this representative fuzzy set (RFS) $\ddot{A}$, given by:

$$
\ddot{A}=\sum_{x \in X} u^{c}
$$

Since RFS provide good representation for T2FS as T1FS, we can use it in the calculation of subsethood.

2) Interval Type-2 Fuzzy Sets: In case of both $\tilde{A}$ and $\tilde{B}$ are interval T2FS, all the secondary grades are constant 1 . So Eq. (17) can be reduced to:

$$
u^{c}=\frac{\underline{u}+\bar{u}}{2}
$$

where $\underline{u}$ and $\bar{u}$ are the upper and lower bounds of primary memberships. Furthermore, the generated RFS will be a T1FS.

From Eq. (18), we write $\ddot{A}=\sum_{x \in X}\left(u^{c}\right)$. For another set $\tilde{B}$ with secondary variable $v$, we also can find a RFS $\ddot{B}=$ $\sum_{x \in X}\left(v^{c}\right)$
Since both $\ddot{A}$ and $\ddot{B}$ are T1FS, we can substitute them into Eq. (6) to get subsethood measurement for $\tilde{A}$ and $\tilde{B}$ :

$$
\pi(\tilde{A} \subseteq \tilde{B})=\bigwedge_{x \in X}\left(u^{c} \rightarrow v^{c}\right)
$$

Please recall that $\rightarrow$ is fuzzy implication operator and $\wedge$ can be considered as function min or arithmetic mean. To make the solution more robust, we consider all the contribution of each pair of elements in $\tilde{A}$ and $\tilde{B}$ and substitute $\wedge$ as arithmetic mean:

$$
\pi(\tilde{A} \subseteq \tilde{B})=\frac{1}{N} \sum_{x \in X}\left(u^{c} \rightarrow v^{c}\right)
$$

where $N$ represents the cardinality.

As mentioned earlier, intervals provide better measurements for the subsethood of T2FS compared to point-values. Equation sets developed in Section III-B provided a framework of forming these T1FS. Eq. (10) and Eq. (13) provide a bound for a measurement, whereas Eq. (16) provides the expected value. By substitute Eq. (21) into these equations, we can easily get an interval for the subsethood of $\tilde{A}$ in $\tilde{B}$ :

$$
\pi(\tilde{A} \subseteq \tilde{B})=\left[s u b_{l}, s u b_{u}\right]
$$

where $s u b_{l}$ and $s u b_{u}$ is the lower and upper bounds of the interval, defined as:

$$
\begin{gathered}
s u b_{l}=\frac{1}{N} \sum_{x \in X} \max \left(v^{c}, 1-u^{c}\right) \\
s u b_{u}=\frac{1}{N} \sum_{x \in X} \min \left(1,1-u^{c}+v^{c}\right)
\end{gathered}
$$

If a single-point value is preferred, the expected value should be:

$$
\pi\left(\tilde{A} \subseteq_{\mathrm{ex}} \tilde{B}\right)=\frac{1}{N} \sum_{x \in X}\left(1-u^{c}+u^{c} v^{c}\right)
$$

The value of $\pi\left(\tilde{A} \subseteq_{\text {ex }} \tilde{B}\right)$ should sit around the middle of the interval $\left[s u b_{l}, s u b_{u}\right]$, so we can denote is as $s u b_{m}$.

3) General Type-2 Fuzzy Sets: In case of the fuzzy sets $\tilde{A}$ and $\tilde{B}$ are general T2FS, the corresponding RFS are embedded T2FS. The main different of these RFS compared to the RFS of interval T2FS is, for each secondary variable $u^{c}$ or $v^{c}$, there is a corresponding secondary grade $f_{x}\left(u^{c}\right)$ or $g_{x}\left(v^{c}\right)$ associated and these secondary grades may not be 1 .

The easiest way of dealing with these secondary grades is to take a strict 0 -cut at all the secondary grades. This can convert all RFS to T1FS, instead of embedded T2FS. Then, the techniques that we used on interval T2FS can be applied here to generate an interval to represent the subsethood measurement. However, this discarded all the information associates with those secondary grades.

Therefore, in order to take into account the secondary grades, we relate them to the membership functions of the intervals (or we should call them sets now) that generated. 


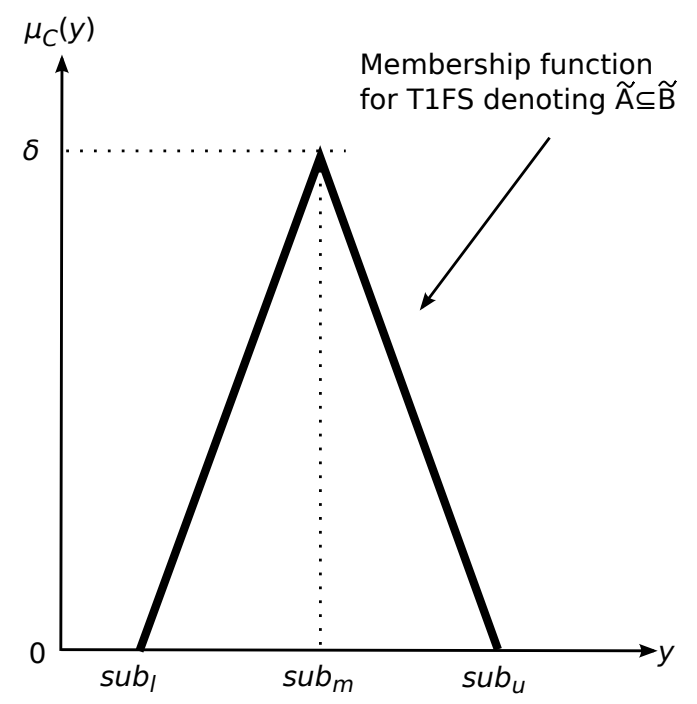

Fig. 2. Obtain a T1FS with triangular membership function with the calculating of $\delta$

Assume that $C \subseteq Y$ is the T1FS that representing subsethood measure $\tilde{A} \subseteq \tilde{B}, \delta$, the height of $C$, is given by:

$$
\delta=\operatorname{Hgt}(C)=\min (\operatorname{Hgt}(\ddot{A}), \operatorname{Hgt}(\ddot{B}))
$$

$\delta$ is the min of the height of both sets, which provides information about the maximum possibility of the measurement. It is reasonable to adopt it as the membership functions of the mid point of the measurement sets:

$$
\mu_{C}\left(s u b_{m}\right)=\delta
$$

and $\mu_{C}\left(s u b_{l}\right)=\mu_{C}\left(s u b_{u}\right)=0$ to form a triangular membership function (Figure 2).

The assignment in Eq. (26) meets the boundary conditions of interval T2FS. If all the $f_{x}\left(u^{c}\right)$ and $g_{x}\left(v^{c}\right)$ are $1, \delta=1$. This is equivalent to the case of both $\tilde{A}$ and $\tilde{B}$ are interval T2FS, and the result of the measurement also return to an interval, rather then a set. When the $f_{x}\left(u^{c}\right)$ and $g_{x}\left(v^{c}\right)$ start decreasing, $\delta=1$ is not hold anymore, and this results in non unity membership functions for $s u b_{l}$ and $s u b_{r}$.

4) Variants of Representative Method: Besides center-ofarea defuzzification, mean-of-maximum defuzzification discussed in [25] may be used to replace center-of-areas to obtain RFS if the involved T2FS are not interval T2FS. A mean-ofmaximum is defined as the mean of all the secondary variables with maximum secondary grades:

$$
u^{m}=\sum_{u \in G_{x}} u /|G|
$$

$G_{x} \subseteq J_{x}$ is the subset where all $u$ with maximum $f_{x}(u)$ in $J_{x}$, and $|G|$ is the cardinality of $G_{x}$. Substituting $u^{c}$ with $u^{m}$ and $v^{c}$ with $v^{m}$ brings a variant to Representative Method. If the sets involved are interval T2FS, one may find that $u^{m}=u^{c}$.
The advantage of using mean-of-maximum over centerof-area is its low computational cost, especially when the secondary membership function is convex. For a system that need simple and fast approximate subsethood measurement, mean-of-maximum always can be used. However, sacrifice of a lot of details is always the cost of this kind of method. Besides, the boundary condition that hold in the case of center-of-sets may not hold with mean-of-maximum.

Karnik and Mendel [27] have developed centroids for T2FS, which is a T1FS. These T2FS centroids provide good representation of their original T2FS in many aspects. However, using T2FS centroids in this Representative Method is not recommended because these centroids do not bring representative at element level. In another words, the generated T2FS centroids will have different domains compare to the original T2FS.

\section{B. Examples}

1) Interval T2FS: Assume that $\tilde{A}$ and $\tilde{B}$ are 2 interval T2FS in universe $X=\left\{x_{1}, x_{2}, \cdots, x_{8}\right\}$. The secondary membership functions of both sets are intervals defined as below:

$$
\begin{array}{r}
\tilde{A}=\{[0.0,0.2],[0.1,0.3],[0.2,0.4],[0.4,0.7],[0.5,1.0], \\
[0.6,0.9],[0.4,0.7],[0.3,0.5]\}
\end{array}
$$

and

$$
\begin{array}{r}
\tilde{B}=\{[0.0,0.3],[0.3,0.5],[0.5,0.7],[0.7,1.0],[0.8,0.9] \\
[0.5,0.8],[0.3,0.6],[0.2,0.4]\}
\end{array}
$$

Using Representation Method, 2 RFS $\ddot{A}$ and $\ddot{B}$ can be generated with finding and linking all corresponding centerof-areas. With Eq. (10) and (13), we can find the interval of approximate subsethood measure $\tilde{A} \subseteq \tilde{B}$ are $[0.725,0.963]$. The expected value for this measurement generated by Eq. (16) is 0.829 .

2) General T2FS: Let $\tilde{A}$ and $\tilde{B}$ are 2 interval T2FS in universe $X=\left\{x_{1}, x_{2}, x_{3}\right\}$. The secondary membership function of each element is defined as a triangular as follow:

$$
\begin{aligned}
& f_{x_{1}}(u)= \begin{cases}(u-0.4) / 0.3 & \text { if } 0.4 \leq u \leq 0.7, \\
(1.0-u) / 0.3 & \text { if } 0.7<u \leq 1.0, \\
0 & \text { otherwise }\end{cases} \\
& f_{x_{2}}(u)= \begin{cases}(u-0.2) / 0.2 & \text { if } 0.2 \leq u \leq 0.4, \\
(0.7-u) / 0.3 & \text { if } 0.4<u \leq 0.7, \\
0 & \text { otherwise }\end{cases} \\
& f_{x_{3}}(u)= \begin{cases}(u-0.4) / 0.3 & \text { if } 0.4 \leq u \leq 0.7, \\
(0.8-u) / 0.1 & \text { if } 0.7<u \leq 0.8, \\
0 & \text { otherwise }\end{cases}
\end{aligned}
$$




$$
\begin{aligned}
& g_{x_{1}}(v)= \begin{cases}(v-0.1) / 0.3 & \text { if } 0.1 \leq v \leq 0.4, \\
(0.5-v) / 0.1 & \text { if } 0.4<v \leq 0.5, \\
0 & \text { otherwise }\end{cases} \\
& g_{x_{2}}(v)= \begin{cases}(v-0.4) / 0.2 & \text { if } 0.4 \leq v \leq 0.6, \\
(0.9-v) / 0.3 & \text { if } 0.6<v \leq 0.9, \\
0 & \text { otherwise }\end{cases} \\
& g_{x_{3}}(v)= \begin{cases}(v-0.2) / 0.5 & \text { if } 0.2 \leq v \leq 0.7, \\
(0.9-v) / 0.2 & \text { if } 0.7<v \leq 0.9, \\
0 & \text { otherwise }\end{cases}
\end{aligned}
$$

In order to solve the problem with Representative Method, we must find all the center-of-areas with Eq. (17) to form $\ddot{A}$ and $\ddot{B}$. Since the secondary membership functions are continuous instead of discrete, we replace $\sum$ in Eq. (17) with $\int$. We should find $\ddot{A}=\{1.00 / 0.70,0.89 / 0.43,0.78 / 0.63\}$ and $\ddot{B}=\{0.78 / 0.33,0.89 / 0.63,0.80 / 0.60\}$. With Eq. (23) - (25), we can find $s u b_{l}=0.520, s u b_{m}=0.710$ and $s u b_{u}=0.870$. For the calculation of membership function of subsethood measure, we use Eq. (26) to find $\delta=0.780$. So, the set representing the subsethood measure $\tilde{A} \subseteq \tilde{B}$ is a triangle:

$$
\mu_{C}(y)= \begin{cases}(y-0.520) / 0.244 & \text { if } 0.520 \leq y \leq 0.710 \\ (0.870-y) / 0.205 & \text { if } 0.710<y \leq 0.870 \\ 0 & \text { otherwise }\end{cases}
$$

\section{CONCLUSion}

We pointed out an important but unattended property of fuzzy sets in this paper, namely multi descriptions property. The property has direct impact on many basic operations of fuzzy sets, such as union, intersection and subsethood.

The popular min and $\max$ functions as measurement of intersection and union in both T1FS and T2FS may bring incomplete results in the present of the multi description property. With Checklist Paradigm, the problem can be solved easily by adopting algebraic product and algebraic sum as replacements in both t-norm and t-conorm.

For subsethood measurement, Bandler and Kohout's definitions which based on implication operators were recalled to solve the problem of T1FS. We extended the solutions to T2FS with Representative Method.

Although the multi descriptions property is not present on all fuzzy sets, but adopting the concern on it may help in developing more general fuzzy systems.

\section{REFERENCES}

[1] G. J. Klir and B. Yuan, Fuzzy Sets And Fuzzy Logic: Theory And Applications. New Jersey: Prentice Hall, 1995.

[2] S. Coupland, "Geometric type-1 and type-2 fuzzy logic systems," Fuzzy Systems, IEEE Transactions on, vol. 15, no. 1, pp. 3-15, 2007.
[3] J. M. Mendel and D. Wu, "Perceptual Reasoning for Perceptual Computing," IEEE Transactions on Fuzzy Systems, vol. 16, no. 6, pp. 15501564, Dec. 2008.

[4] B. Kosko, "Fuzzy entropy and conditioning," Information Sciences, vol. 40 , no. 2 , pp. $165-174,1986$.

[5] L. J. Kohout and W. Bandler, "Semantics of implication operators and fuzzy relational products," International Journal of Man-Machine Studies, vol. 12, no. 1, pp. 89-116, Jan. 1980.

[6] H. Nguyen and V. Kreinovich, "Computing Degrees of Subsethood and Similarity for Interval-Valued Fuzzy Sets : Fast Algorithms," in Proceedings of the 9th International Conference on Intelligent Technologies, Samui, Thailand, 2008, pp. 47-55.

[7] M.-S. Yang and D.-C. Lin, "On similarity and inclusion measures between type-2 fuzzy sets with an application to clustering," Computers \& Mathematics with Applications, vol. 57, no. 6, pp. 896-907, Mar. 2009.

[8] G. Zheng, J. Xiao, Y. Zhang, and G. Shi, "An inclusion measure between general type-2 fuzzy sets," in Fuzzy Systems and Knowledge Discovery (FSKD), 2010 Seventh International Conference on, vol. 1, aug. 2010, pp. $99-103$.

[9] L. A. Zadeh, "The concept of a linguistic variable and its application to approximate reasoning-I," Information Sciences, vol. 8, no. 3, pp. 199-249, 1975.

[10] M. Mizumoto and K. Tanaka, "Some properties of fuzzy sets of type 2," Information and Control, vol. 31, no. 4, pp. 312-340, Aug. 1976.

[11] J. M. Mendel, "Type-2 Fuzzy Sets And Systems: An Overview," IEEE Computational Intelligence Magazine, vol. 20, no. 3, pp. 20-29, Mar. 2007.

[12] J. M. Mendel and R. John, "Type-2 fuzzy sets made simple," IEEE Transactions on Fuzzy Systems, vol. 9, no. 2, p. 55, Apr. 2007.

[13] L. J. Kohout and W. Bandler, "Fuzzy Power Sets And Fuzzy Implication Operators," Fuzzy Sets and Systems, vol. 4, pp. 13-30, 1980.

[14] R. Willmott, "Two fuzzier implication operators in the theory of fuzzy power sets," Fuzzy Sets and Systems, vol. 4, no. 1, pp. 31 - 36, 1980.

[15] D. Wu and J. Mendel, "Perceptual reasoning for perceptual computing: A similarity-based approach," Fuzzy Systems, IEEE Transactions on, vol. 17, no. 6, pp. 1397-1411, Dec. 2009.

[16] D. Wu and J. M. Mendel, "Computing with words for hierarchical decision making applied to evaluating a weapon system," Fuzzy Systems, IEEE Transactions on, vol. 18, no. 3, pp. 441-460, Jun. 2010.

[17] $\mathrm{D}$. Wu and J. Mendel, "Linguistic summarization using if-then rules and interval type-2 fuzzy sets," Fuzzy Systems, IEEE Transactions on, vol. 19 , no. 1, pp. $136-151$, feb. 2011.

[18] L. J. Kohout and W. Bandler, "Relational-Product Architectures for Information Processing," Information Sciences, vol. 37, pp. 25-37, 1985.

[19] B. De Baets and E. Kerre, "Fuzzy relational compositions," Fuzzy Sets and Systems, vol. 60, no. 1, pp. 109-120, Nov. 1993.

[20] J. T. Rickard, J. Aisbett, and G. Greb, "Fuzzy Subsethood for Fuzzy Sets of Type-2 and Generalized Type-n," IEEE Transactions on Fuzzy Systems, vol. 17, no. 1, pp. 50-60, Feb. 2009.

[21] B. Kosko, "Fuziness vs. Probability," International Journal of General Systems, vol. 17, pp. 211-240, 1990.

[22] D. Wu and J. M. Mendel, "Efficient algorithms for computing a class of subsethood and similarity measures for interval type-2 fuzzy sets," in Fuzzy Systems (FUZZ), 2010 IEEE International Conference on, no. 7. IEEE, 2010, pp. 1-7.

[23] C. C. Lee, "Fuzzy logic in control systems: fuzzy logic controller, part II," IEEE Transactions on Systems, Man, and Cybernetics, vol. 20, no. 2, pp. 419-435, 1990.

[24] N. N. Karnik, J. M. Mendel, and Q. Liang, "Type-2 Fuzzy Logic Systems," IEEE Transactions on Fuzzy Systems, vol. 7, no. 6, pp. 643658, 1999.

[25] D. P. Filev and R. R. Yager, "A generalized defuzzification method via bad distributions," International Journal of Intelligent Systems, vol. 6, no. 7, pp. 687-697, Dec. 1991

[26] J. Castro, "Fuzzy logic controllers are universal approximators," IEEE Transactions on Systems, Man, and Cybernetics, vol. 25, no. 4, pp. 629635, Apr. 1995.

[27] N. Karnik and J. M. Mendel, "Centroid of a type-2 fuzzy set," Information Sciences, vol. 132, no. 1-4, pp. 195-220, Feb. 2001. 